\title{
Activating transcription factor 3 is crucial for antitumor activity and to strengthen the antiviral properties of Onconase
}

\author{
Anna Vert ${ }^{1,2}$, Jessica Castro ${ }^{1,2}$, Marc Ribó ${ }^{1,2}$, Antoni Benito ${ }^{1,2}$, Maria Vilanova ${ }^{1,2}$ \\ ${ }^{1}$ Laboratori d'Enginyeria de Proteïnes, Departament de Biologia, Facultat de Ciències, Universitat de Girona, Campus de \\ Montilivi, 17003, Girona, Spain \\ ${ }^{2}$ Institut d'Investigació Biomèdica de Girona Josep Trueta, (IdIBGi), Girona, Spain \\ Correspondence to: Maria Vilanova, email: maria.vilanova@udg.edu \\ Antoni Benito, email: antoni.benito@udg.edu
}

Keywords: antitumor and antiviral drug, Onconase, activating transcription factor 3, microarray profiling, apoptosis

Received: June 04, 2016

Accepted: November 30, 2016

Published: December 27, 2016

\section{ABSTRACT}

Onconase is a ribonuclease that presents both antitumor and antiviral properties linked to its ribonucleolytic activity and represents a new class of RNA-damaging drugs. It has reached clinical trials for the treatment of several cancers and human papilloma virus warts. Onconase targets different RNAs in the cell cytosol but Onconase-treated cells present features that are different from a simple arrest of protein synthesis. We have used microarray-derived transcriptional profiling to identify Onconase-regulated genes in two ovarian cancer cell lines (NCI/ADR-RES and OVCAR-8). RT-qPCR analyses have confirmed the microarray findings. We have identified a network of up-regulated genes implicated in different signaling pathways that may explain the cytotoxic effects exerted by Onconase. Among these genes, activating transcription factor 3 (ATF3) plays a central role in the key events triggered by Onconase in treated cancer cells that finally lead to apoptosis. This mechanism, mediated by ATF3, is cell-type independent. Up-regulation of ATF3 may also explain the antiviral properties of this ribonuclease because this factor is involved in halting viral genome replication, keeping virus latency or preventing viral oncogenesis. Finally, Onconase-regulated genes are different from those affected by nucleardirected ribonucleases.

\section{INTRODUCTION}

Onconase (ONC), a protein of amphibian origin, is a member of the vertebrate secreted ribonucleases (RNases) family that presents antitumor and antiviral activities. Its structure endows it with unusual high conformation stability [1] and with the ability to evade the cytoplasmic ribonuclease inhibitor (RI), a $50 \mathrm{kDa}$ protein that tightly binds to some RNases and inhibits their activity [2]. Both its conformational stability and its ability to evade the RI provide ONC with significant intracellular survival that is critical to its biological actions.

Regarding the antitumor activity, it has been shown that $\mathrm{ONC}$ exhibits selective cytostatic and cytotoxic activities [3] against many human tumor models (for a review see $[4,5])$. In addition, ONC presents synergy with a significant number of anti-tumor drugs, such as tamoxifen, lovastatin, cisplatin, and vincristine, among others (for a review see [6]). More interestingly, it has entered clinical trials for the treatment of different types of cancer and has reached phase II/III for the treatment of malignant mesothelioma [7-9].

The ONC-induced apoptotic effects linked to its cell internalization mechanism are still not well understood. It is believed that the normal route for ONC to reach the cytosol is through an endosomal compartment, but controversy exists about the existence of a cellular receptor [6]. Once in the cytosol, ONC degrades RNA, thereby preventing protein synthesis and inducing cell cycle arrest and apoptosis. However, ONC-induced apoptosis presents features different from those of indiscriminate protein synthesis arrest (for a review see [6]). In this regard, it has been demonstrated that, besides rRNA, the initially proposed ONC target [10], mRNAs [11, 12], tRNAs [13], and miRNAs or their precursors [14-16] are also ONC targets. Accordingly, ONC up- and down-regulates genes that code for proteins involved in cell cycle progression or transcription factors involved in cell survival $[11,12,17]$. 
In addition, ONC may trigger cell death in several ways, depending on the cell type. It may induce caspase-dependent apoptosis linked to the activation of the stress-activated protein kinase c-Jun NH2 [18] or intrinsic apoptosis together with the activation of Ser-proteases [19, 20]. It has been demonstrated that intrinsic apoptosis depends on Apaf1 reversing the inhibitory effect of tRNAs on the association of cytochrome c with Apaf-1 [21]. In addition, ONC may induce cell death by autophagy $[22,23]$.

The antiviral activity of ONC is of particular interest due to ONC's ability to degrade not only RNA but dsRNA [24]. Antiviral drugs that destroy viral genomes not only suppress virus production but are likely to abolish latent virus infection. It has been described that ONC exhibits efficacy in suppressing the replication of HIV-1, leaving non-infected cells unharmed [25]. Recently, several patents have been obtained by Tamir Biotechnology, Inc. (San Diego, CA) (http://tamirbio.com/) to use wild type ONC and variants as antiviral agents. The most success has been obtained with human papilloma virus (HPV) [26], although other viral infections, such as those of the Herpesviridae family, are also being treated [27]. ONC is now in clinical trial to study its safety and efficacy by topical administration to treat genital warts (clinical trial identifier: NCT02535104 at https://clinicaltrials. gov/). What is more, ONC is being considered alone or as adjuvant therapy in an effort to fight Ebola, orthomyxoviruses (influenza), Middle East Respiratory Syndrome (MERS-CoV), the Hepatitis C virus (HCV), the cytomegalovirus (CMV), the respiratory syncytial virus (RVS), and rhabodviruses (rabies) (http://tamirbio.com/).

Taking into account the large number of diseases with high social impact that are or can be treated with ONC, we have used microarray technology to identify significant altered gene expression in the treatment of two ovarian carcinoma cell lines, NCI/ADR-RES and its parental OVCAR-8, with this enzyme. The present work is the second to use microarray-derived transcriptional profiling to identify ONC-regulated genes in order to understand the cytotoxic mechanism of this RNase. In a previous work, Altomare et al. [12] investigated the effect of ONC on different human malignant mesothelioma (hMM) cell lines and showed that this RNase alters the expression of genes that control the cell cycle and apoptosis.

Our results show that the ONC up-regulated activating transcription factor 3 (ATF3) is a cell-type independent key factor of ONC antitumor activity. ATF3 activates other transcription factors that control different signaling pathways. This explains the effects observed in the cell cycle and the induction of apoptosis in cells treated with ONC. Interestingly, it has been described that ATF3 and some of its regulated genes help to preclude viral genome replication, to maintain virus latency, or to prevent viral oncogenesis. Finally, our results also show that the pleiotropic effects caused by ONC, derived from its ribonucleolytic activity in the cytosol, are completely different from those promoted by nuclear-directed RNases (ND-RNases) [28].

\section{RESULTS}

ONC is a paradigmatic antitumor RNase whose cytotoxic mechanism has been studied by different means [6]. Nevertheless, microarray profiling to broadly analyze the genes affected by this drug has only been done in hMM cell lines [12]. We have used the same approach to study the effects of ONC in two ovarian carcinoma cell lines, NCI/ADR-RES and its parental OVCAR-8. In addition, our results allow us to compare the effects on gene expression promoted by two different RNases in cancer cells: ONC, an RNase that exerts its action in the cytosol, and PE5, an ND-RNase [29].

\section{Cell proliferation and RNase sensitivity}

Since ONC has ribonucleolytic activity, an unbiased approach to examine gene expression by microarray technology requires searching for optimal cell treatment conditions. An RNA population representative of the actual drug effect must be obtained, not one that results from an extensive RNA degradation caused by the apoptosis process [30]. Therefore, we investigated the cytotoxic effect of ONC on NCI/ADR-RES and OVCAR-8 cells at different $\mathrm{ONC}$ concentrations and incubation times, as we had previously done with PE5 [28]. A dose-dependent cytotoxic effect was apparent after $24 \mathrm{~h}$ of incubation with ONC and increased with time (Supplementary Figure 1 Supplementary Data). As expected [31], the cytotoxic effect of ONC was greater than that of PE5 [28], and the MDR cell line, NCI/ADR-RES, was less sensitive to both RNases than its parental cell line OVCAR-8.

Next, we analyzed the RNA degradation caused by different doses of ONC. We incubated NCI/ADR-RES and OVCAR-8 cells with ONC concentrations that induced a decrease in cell proliferation of $5 \%, 10 \%$, and $15 \%$ $\left(\mathrm{IC}_{5}, \mathrm{IC}_{10}\right.$, and $\left.\mathrm{IC}_{15}\right)$. After $36 \mathrm{~h}$ of incubation, the RNA degradation of treated and untreated cells was quantified using a bioanalyzer. The RNA integrity number (RIN) values obtained for all samples were higher than 9.3. For a good comparison of the effects of ONC and PE5 on the NCI/ADR-RES cell line, we decided to carry out the microarray experiments at that concentration of $\mathrm{ONC}$ that triggered the same decrease in cell viability $(10 \%)$ observed with PE5 [28] after $36 \mathrm{~h}$ of treatment (i.e. 0.5 $\mu \mathrm{M})$. Likewise, we chose an ONC concentration for the OVCAR-8 cell line of $0.06 \mu \mathrm{M}$, which also produced a $10 \%$ decrease in cell proliferation.

\section{Gene expression changes in ONC-treated cells}

ONC-treated and untreated NCI/ADR-RES cells revealed 56 differentially expressed genes out of 35,377 
present in the microarray $\left(1.6 \times 10^{-3} \%\right)$. Among them, $89 \%$ were up-regulated (the increase from untreated cells ranged from 2 - to 6.5 -fold), whereas $11 \%$ were down-regulated (the decrease from untreated cells ranged from 2- to 2.5-fold). Table 1 shows the top 20 ONC upregulated genes as well as all the ONC down-regulated genes. The results indicate that the primary effect of ONC in NCI/ADR-RES cells is the activation of gene expression. ONC up-regulated genes are involved in transcription regulation (ATF3, CREB5, EGR1), cell cycle and apoptosis (GADD45A, PPP1R15A), immune response (IL6, IL33, IL1RL1, IL23A), and stress response (TXNIP), among other processes. Some of the upregulated genes act as tumor suppressors (TXNIP, EGR1, and PPP1R15A). ONC down-regulated genes are also associated with transcription regulation (RDBP, XBP1), amino acid metabolism (PHGDH, ASNS), and protein folding (HSPA1A, HSPA8).

ONC-treated and untreated OVCAR- 8 cells revealed seven differentially expressed genes out of 35,377 present in the microarray $\left(1.8 \times 10^{-4} \%\right)$. All of them were upregulated (the increase from untreated cells ranged from 2- to 4-fold) (Table 2), indicating that the primary effect of $\mathrm{ONC}$ is also gene expression activation. Again, some of the up-regulated genes are related to transcription regulation (ATF3, NEURL3, ZNF750), while others are involved in stress response (TXNIP). Six out of the seven ONC differentially expressed genes in the OVCAR-8 cell line were also differentially expressed in the NCI/ADRRES cell line (TXNIP, ATF3, HIST1H2AC, NEURL3, DHRS2, and NPPB).

Among the ONC and PE5 differentially expressed genes in the NCI/ADR-RES cell line, we identified only 10 that were common for both RNase treatments (i.e. 1.5\% and $18 \%$ of the PE5 [28] and ONC differentially expressed genes, respectively). Eight of them were up-regulated in RNase-treated cells respective to untreated cells, whereas the other two were down-regulated. Common up-regulated genes are related to transcription regulation (HMBOX1, LINC00340, NEXN-AS1), immune response (CD86, IL6, IFIT2) and the last two are potential tumor suppressors (LRRC2 and SPINK6 [32, 33]). Common down-regulated genes participate in amino acid metabolism (PHGDH, ASNS).

\section{Quantitative real-time PCR analysis of gene expression}

mRNA expression of three ONC up-regulated (TXNIP, ATF3, and GADD45A) and two down-regulated (PHGDH, ASNS) genes, representatives of the processes affected by this RNase, was analyzed by RT-qPCR. The results are presented in Supplementary Figure 2 of the Supplementary Data. Fold changes obtained with RTqPCR, calculated as the ratio between relative transcript abundance (RTA) values obtained for ONC-treated cells and RTA values obtained for untreated cells, were similar to those found in the microarray analysis (Figure 1), confirming that the microarray experiments are fully valid.

\section{Gene ontology analysis and KEGG pathway annotation}

To better understand the functional relevance of ONC-regulated genes in NCI/ADR-RES cells, we performed a gene ontology analysis. To the same end, ONC differentially expressed genes were mapped to the KEGG database to find over-represented known metabolic and regulatory pathways. These analyses were not done for the OVCAR-8 cell line because ONC-treatment gave only 10 differentially expressed genes. The NCI/ ADR-RES differentially expressed genes were used to find over-represented gene ontology terms in the three broad ontology categories: "molecular function", which captures knowledge about the functional activity of gene products, the larger "biological process", as part of which these specific functions collectively act, and "cellular component", where all this occurs. Gene ontology analysis shows that ONC differentially expressed genes (Table 3) are involved in a diversity of cellular events. The same was found for PE5, showing that these RNA-damaging drugs have pleiotropic effects [28].

The most ONC-affected biological processes were cell proliferation, response to stress, signal transduction, cell differentiation, and apoptosis. The individual function of ONC-regulated genes favored cytokine activity, nucleic acid binding transcription factor activity, and growth factor activity, and were mainly associated with the extracellular region, the cell surface, and the nucleus.

Analysis of the over-represented pathways collected in the KEGG database showed that ONCaffected pathways (Table 3 ) were involved in growth and development processes, like JAK-STAT or the MAPK signaling pathway, which affect basic cellular functions such as cell growth, proliferation, differentiation, and death, as well as stress response. Some pathways related to infection or immunological diseases were also affected.

\section{DISCUSSION}

\section{ONC cytotoxic mechanism}

Gene ontology analysis and KEGG pathway annotation of ONC-differentially expressed genes in the NCI/ADR-RES cell line show interesting terms that allow us to understand how ONC induces cell death. These terms are cell proliferation, response to stress, and apoptotic processes (Table 3). In these fields we can find 7 of the 20 most ONC up-regulated genes in NCI/ADRRES cells (Table 1) that correspond to functionally related transcription regulators: early growth response 1 (EGR1), activating transcription factor 3 (ATF3), growth arrest and 
Table 1: ONC differentially expressed genes in NCI/ADR-RES cell line: top 20 ONC up-regulated genes and the 6 down-regulated genes

\begin{tabular}{|c|c|c|c|c|}
\hline Probe ID & Gene Symbol & Gene Name & Fold Change & Main Functions \\
\hline A_23_P97700 & TXNIP & Thioredoxin interacting protein & 6.5 & $\begin{array}{c}\text { Transcription regulation } \\
\text { Cell cycle } \\
\text { Cell proliferation } \\
\text { Stress response } \\
\text { Apoptosis }\end{array}$ \\
\hline A_23_P34915 & ATF3 & Activating transcription factor 3 & 6.5 & Transcription regulation \\
\hline A_23_P157117 & CREB5 & $\begin{array}{l}\text { cAMP responsive element binding } \\
\text { protein } 5\end{array}$ & 6.2 & Transcription regulation \\
\hline A_23_P167983 & HIST1H2AC & Histone cluster $1, \mathrm{H} 2 \mathrm{ac}$ & 4.0 & Nucleosome assembly \\
\hline A_23_P339818 & ARRDC4 & Arrestin domain containing 4 & 3.9 & Signal transduction \\
\hline A_23_P328740 & NEURL3 & $\begin{array}{c}\text { Neuralized homolog } 3 \text { (Drosophila) } \\
\text { pseudogene }\end{array}$ & 3.6 & Transcription regulation \\
\hline A_23_P23221 & GADD45A & $\begin{array}{l}\text { Growth arrest and DNA-damage- } \\
\text { inducible, alpha }\end{array}$ & 3.2 & $\begin{array}{l}\text { Cell cycle } \\
\text { DNA repair } \\
\text { Apoptosis }\end{array}$ \\
\hline A_23_P71037 & IL6 & Interleukin 6 (interferon, beta 2) & 3.2 & $\begin{array}{c}\text { Immune response } \\
\text { Cell differentiation } \\
\text { Cell proliferation } \\
\text { Apoptosis } \\
\text { Transcription regulation }\end{array}$ \\
\hline A_23_P259071 & AREG & Amphiregulin & 3.2 & Cell proliferation \\
\hline A_23_P92909 & SPINK6 & $\begin{array}{c}\text { Serine peptidase inhibitor, Kazal } \\
\text { type } 6\end{array}$ & 3.0 & Epidermis development \\
\hline A_23_P214080 & EGR1 & Early growth response 1 & 3.0 & Transcription regulation \\
\hline A_23_P90172 & PPP1R15A & $\begin{array}{c}\text { Protein phosphatase } 1 \text {, regulatory } \\
\text { subunit } 15 \mathrm{~A}\end{array}$ & 2.7 & $\begin{array}{c}\text { Apoptosis } \\
\text { Stress response } \\
\text { Translation regulation } \\
\text { Cell cycle }\end{array}$ \\
\hline A_32_P18440 & ARID5B & $\begin{array}{l}\text { AT rich interactive domain 5B } \\
\text { (MRF1-like) }\end{array}$ & 2.5 & Transcription regulation \\
\hline A_32_P141238 & ANO2 & Anoctamin 2 & 2.5 & Ion transport \\
\hline A_23_P139500 & BHLHE41 & $\begin{array}{l}\text { Basic helix-loop-helix family, } \\
\text { member e41 }\end{array}$ & 2.5 & Transcription regulation \\
\hline A_23_P321501 & DHRS2 & $\begin{array}{l}\text { Dehydrogenase/reductase (SDR } \\
\text { family) member } 2\end{array}$ & 2.5 & Stress response \\
\hline A_23_P320739 & MEF2C & Myocyte enhancer factor $2 \mathrm{C}$ & 2.5 & $\begin{array}{c}\text { Transcription regulation } \\
\text { Cell differentiation } \\
\text { Immune response }\end{array}$ \\
\hline A_33_P3243887 & IL11 & Interleukin 11 & 2.4 & $\begin{array}{l}\text { Cell differentiation } \\
\text { Cell proliferation } \\
\text { Transcription regulation }\end{array}$ \\
\hline A_24_P932736 & HMBOX1 & Homeobox containing 1 & 2.4 & Transcription regulation \\
\hline A_23_P51126 & IL1RL1 & Interleukin 1 receptor-like 1 & 2.4 & Immune response \\
\hline
\end{tabular}

(Continued) 


\begin{tabular}{|c|c|c|c|c|}
\hline Probe ID & Gene Symbol & Gene Name & Fold Change & Main Functions \\
\hline A_33_P3390758 & HSPA8 & Heat shock $70 \mathrm{kDa}$ protein 8 & -2.0 & $\begin{array}{c}\text { Protein folding } \\
\text { Unfolded protein response }\end{array}$ \\
\hline A_23_P120845 & XBP1 & $\mathrm{X}$-box binding protein 1 & -2.2 & $\begin{array}{l}\text { Transcription regulation } \\
\text { Unfolded protein response }\end{array}$ \\
\hline A_23_P111132 & HSPA1A & Heat shock $70 \mathrm{kDa}$ protein $1 \mathrm{~A}$ & -2.3 & $\begin{array}{c}\text { Protein folding } \\
\text { Unfolded protein response }\end{array}$ \\
\hline A_23_P145694 & ASNS & $\begin{array}{c}\text { Asparagine synthetase (glutamine- } \\
\text { hydrolyzing) }\end{array}$ & -2.3 & $\begin{array}{l}\text { Amino acid metabolism } \\
\text { Unfolded protein response }\end{array}$ \\
\hline A_23_P85783 & PHGDH & Phosphoglycerate dehydrogenase & -2.5 & Amino acid metabolism \\
\hline A_23_P122545 & RDBP & RD RNA binding protein & -2.5 & Transcription regulation \\
\hline
\end{tabular}

Gene information was taken from the UniProt database (European Bioinformatics, UK, Swiss Institute of Bioinformatics, Switzerland, Protein Information Resource, USA) (http://www.uniprot.org) and from the Entrez Gene database (National Center for Biotechnology Information, USA) (http://www.ncbi.nlm.nih.gov/gene).

DNA-damage-inducible, alpha (GADD45A), thioredoxin interacting protein (TXNIP), cAMP responsive element binding protein 5 (CREB5), and protein phosphatase 1, regulatory subunit 15A (PPP1R15A). Their relationship is depicted in Figure 2.

ATF3 is a member of the ATF/CREB family of transcription factors that transduces signals from various receptors to activate or repress gene expression. Depending on the cell type and context it plays different roles in cancer development [34]. Nevertheless, its ectopic expression induces apoptosis in a variety of cancer cells $[35,36]$, enhances the ability of different drugs, such as camptothecin, etoposide, curcumin, cisplatin and lovastatin, to induce apoptosis [37-40], and even inhibits the invasion and metastasis of cancer cells [41-43]. ATF3 was first described as associated with ONC activity by Altomare et al. [12]. Our results seem to indicate that it plays a central role in the ONC action because it directly up-regulates the expression of GADD45A [44] and PPP1R15A [45]. Additionally, it can indirectly increase TXNIP expression, since it promotes up-regulation of the Krüppel-like factor 6 (KLF6) [44], a transcription factor whose overexpression in turn increases that of TXNIP [46]. KLF6 is also up-regulated by ONC, although the fold change is lower than 2. Moreover, ATF3 can bind to CREB5 to form a heterodimer [47]. Although the physiological significance of this dimer is not clear, the general view is that its formation can alter DNA binding specificity and transcriptional activity, thus expanding the ability of this transcription factor to regulate gene expression [34]. Finally, it is described that the expression of ATF3 [48] and GADD45A [49] is increased by EGR1 (Figure 2). EGR1 is expressed at low levels in several types of cancer [50-52], acting as a tumor suppressor and inducing apoptosis of cancer cells in a p53-independent manner [53]. It is interesting to note that ONC exerts its cytotoxicity independently of the p53 phenotype [54].
GADD45A and PPP1R15A belong to the family of growth arrest and DNA damage (GADD) proteins, which are crucial to cellular stress responses [55]. It is known that GADD45A inhibits cell growth through the $\mathrm{G}_{2} / \mathrm{M}$ cell cycle arrest $[56,57]$ while PPP1R15A plays a vital role in promoting cell death following proteasome inhibition via enhancing protein synthesis to activate mechanisms associated with dead, including ER stress, ROS production, and autophagy formation [58]. In addition, down-regulation of GADD45A promoted by the NF- $\mathrm{kB}$ transcription factor is essential for cancer cell survival [59]. In this regard, it is worth mentioning that ONC mediates some of its effects by reducing cellular levels of NF-kB. In Jurkat cells, ONC induces an arrest of proliferation, accompanied by an altered subcellular distribution and reduced expression of NF- $\mathrm{KB}$ [17]. In addition, in hMM cells $\mathrm{ONC}$ inhibits NF- $\mathrm{KB}$ nuclear translocation induced by TNF- $\alpha$ [60] and produces a down-regulation of the NFKB1 gene, which encodes the NF- $\kappa \mathrm{B}$ subunit $\mathrm{p} 50$, mediated by the increase in hsamiR-17* and the decrease in hsa-miR-30c levels [61]. It can be expected that, in these cell lines, the inactivation of NF- $\kappa B$ would also lead to the up-regulation of GADD45A.

TXNIP, a protein with multiple functions, plays an important role in redox homeostasis [62]. Extensive evidence shows that TXNIP acts as a tumor suppressor [63-69]. ONC induces the expression of two ATF3controlled genes whose encoded proteins promote cell cycle arrest at different checkpoints (G2/M in the case of GADD45A and G0/G1 in the case of TXNIP $[64,70]$ ). This effect would be in accordance with previous results showing that ONC arrests cell growth of Jurkat and NCI/ ADR-RES cells without altering the percentage of cells in the different cell cycle phases $[17,31]$.

ONC-regulated genes significantly affect the MAPK signaling pathway (Table 3). In this regard, it is noteworthy that TXNIP, through ASK1 activation [71], 
Table 2: ONC differentially expressed genes in OVCAR-8 cell line

\begin{tabular}{|c|c|c|c|c|}
\hline Probe ID & Gene Symbol & Gene Name & Fold Change & Main Functions \\
\hline A_23_P34915 & ATF3 & Activating transcription factor 3 & 4.0 & Transcription regulation \\
\hline A_23_P97700 & TXNIP & Thioredoxin interacting protein & 3.2 & $\begin{array}{c}\text { Transcription regulation } \\
\text { Cell cycle } \\
\text { Cell proliferation } \\
\text { Stress response } \\
\text { Apoptosis }\end{array}$ \\
\hline A_23_P328740 & NEURL3 & $\begin{array}{c}\text { Neuralized homolog } 3 \text { (Drosophila) } \\
\text { pseudogene }\end{array}$ & 2.9 & Transcription regulation \\
\hline A_33_P3423420 & ZNF750 & Zinc finger protein 750 & 2.3 & Transcription regulation \\
\hline A_23_P167983 & HIST1H2AC & Histone cluster $1, \mathrm{H} 2 \mathrm{ac}$ & 2.3 & Nucleosome assembly \\
\hline A_23_P321501 & DHRS2 & $\begin{array}{l}\text { Dehydrogenase/reductase (SDR } \\
\text { family) member } 2\end{array}$ & 2.2 & Stress response \\
\hline A_23_P62752 & NPPB & Natriuretic peptide B & 2.1 & Cardiovascular homeostasis \\
\hline
\end{tabular}

Gene information was taken from the UniProt database (European Bioinformatics, UK, Swiss Institute of Bioinformatics, Switzerland, Protein Information Resource, USA) (http://www.uniprot.org) and from the Entrez Gene database (National Center for Biotechnology Information, USA) (http://www.ncbi.nlm.nih.gov/gene).

and GADD45A, through its interaction with mitogenactivated protein kinase kinase kinase 4 (MEKK4), are involved in the apoptosis induced by the p38 MAPK/JNK signaling pathway [72]. At the same time, the activation of p38 MAPK/JNK induces the expression of EGR1 $[73,74]$ and ATF3 [75], reinforcing the initial action of ONC thorough the up-regulation of these two genes (Figure 3). Accordingly, ONC is a potent activator of the stress-activated protein kinases JNK1, JNK2 and p38 MAPK in primary and immortalized fibroblasts [18].
In this work we show that, in addition to the MAPK pathway, ONC affects the Jak-STAT signaling pathway by altering the expression of different genes. Interestingly, it has been previously described that the cytotoxic effect of ONC on hMM cells is also mediated by the Jak-STAT pathway [12]. Regarding the Jak-STAT signaling, ONC treated NCI/ ADR-RES cells show an increased expression of interleukin 6 (IL6) (Table 1 and Figure 2), previously observed in hMMtreated cells [12]. IL6 is a pleiotropic cytokine that presents both pro- and anti-inflammatory properties as well as pro- and
NCI/ADR-RES

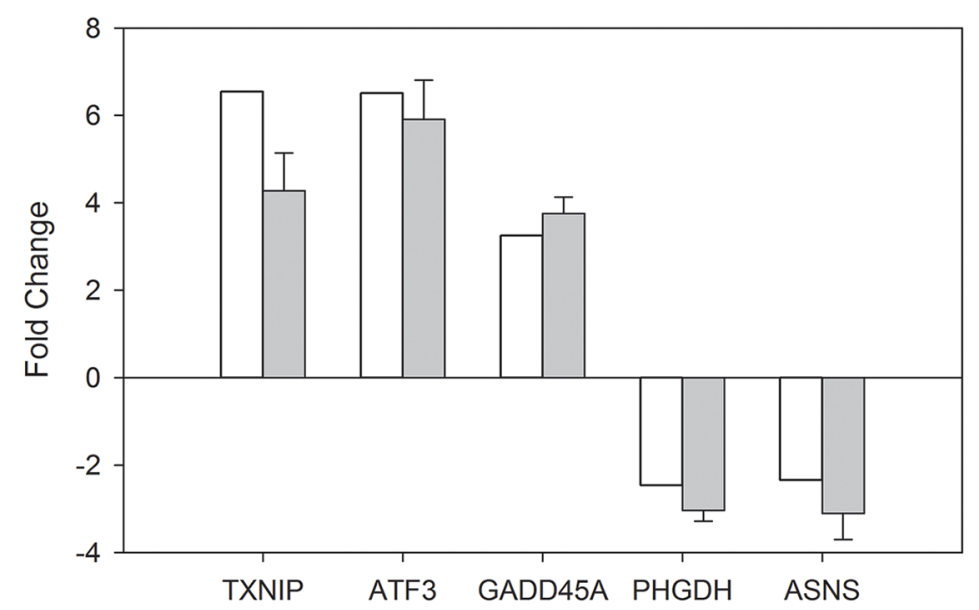

OVCAR-8

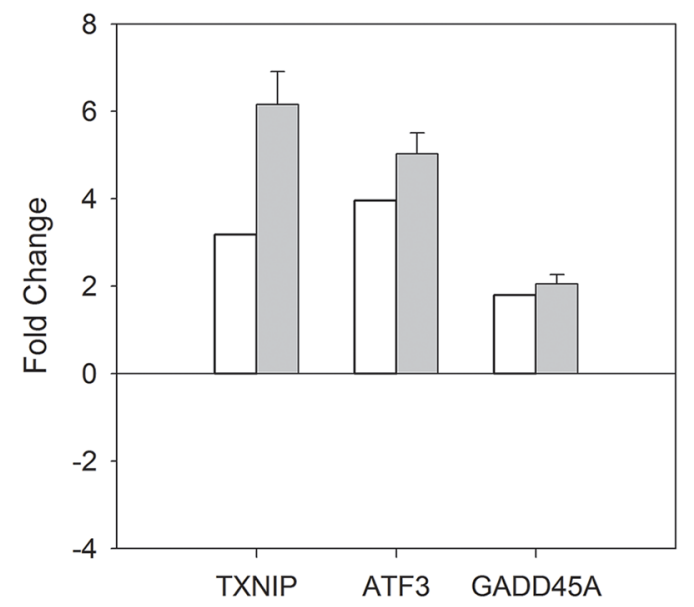

Figure 1: Quantitative comparison of gene expression changes in NCI/ADR-RES and OVCAR-8 cell lines. The histograms show the fold change values of selected genes (gene intensities of treated relative to control samples) obtained by microarrays (white) and RT-qPCR (grey) in NCI/ADR-RES and OVCAR-8 cell lines. RT-qPCR data are presented as mean \pm SD. Genes: Thioredoxin interacting protein (TXNIP), activating transcription factor 3 (ATF3), growth arrest and DNA-damage-inducible, alpha (GADD45A), phosphoglycerate dehydrogenase (PHGDH), and asparagine synthetase (glutamine-hydrolyzing) (ASNS). 
Table 3: Gene ontology analysis and KEGG pathway annotation of ONC differentially expressed genes in NCI/ADRRES cell line

\begin{tabular}{|c|c|c|c|}
\hline Analysis & Term & Gene count $^{\text {a }}$ & P-value \\
\hline \multirow[t]{19}{*}{ Gene Ontology - Biological Process } & Cell proliferation & 15 & $2.45 \mathrm{E}-05$ \\
\hline & Response to stress & 21 & $3.18 \mathrm{E}-05$ \\
\hline & Signal transduction & 24 & $2.03 \mathrm{E}-04$ \\
\hline & Cell differentiation & 18 & 4.34E-04 \\
\hline & Apoptotic process & 13 & $4.81 \mathrm{E}-04$ \\
\hline & Developmental process & 24 & $7.50 \mathrm{E}-04$ \\
\hline & Immune response & 10 & $9.98 \mathrm{E}-04$ \\
\hline & Transcription, DNA-dependent & 18 & $3.44 \mathrm{E}-03$ \\
\hline & Phosphorylation & 10 & $3.95 \mathrm{E}-03$ \\
\hline & Cell-cell signaling & 9 & 4.46E-03 \\
\hline & Chromatin assembly & 3 & $5.46 \mathrm{E}-03$ \\
\hline & Protein metabolic process & 20 & $5.80 \mathrm{E}-03$ \\
\hline & Intracellular protein kinase cascade & 7 & $1.45 \mathrm{E}-02$ \\
\hline & Gene expression & 20 & $2.20 \mathrm{E}-02$ \\
\hline & Angiogenesis & 4 & $2.24 \mathrm{E}-02$ \\
\hline & Growth & 6 & $2.66 \mathrm{E}-02$ \\
\hline & RNA metabolic process & 18 & $2.84 \mathrm{E}-02$ \\
\hline & Cell migration & 6 & $3.94 \mathrm{E}-02$ \\
\hline & DNA metabolic process & 6 & $4.52 \mathrm{E}-02$ \\
\hline \multirow[t]{4}{*}{ Gene Ontology - Molecular Function } & Cytokine activity & 4 & $3.66 \mathrm{E}-03$ \\
\hline & $\begin{array}{l}\text { Nucleic acid binding transcription factor } \\
\text { activity }\end{array}$ & 8 & $1.10 \mathrm{E}-02$ \\
\hline & Growth factor activity & 3 & $1.38 \mathrm{E}-02$ \\
\hline & Hormone activity & 2 & $4.48 \mathrm{E}-02$ \\
\hline \multirow[t]{3}{*}{ Gene Ontology - Cellular Component } & Extracellular region & 15 & $6.05 \mathrm{E}-04$ \\
\hline & Cell surface & 6 & $2.35 \mathrm{E}-03$ \\
\hline & Nucleus & 24 & $2.30 \mathrm{E}-02$ \\
\hline \multirow[t]{9}{*}{ KEGG pathway annotation } & Prion diseases & 3 & $4.59 \mathrm{E}-04$ \\
\hline & Rheumatoid arthritis & 4 & $6.22 \mathrm{E}-04$ \\
\hline & MAPK signaling pathway & 6 & $9.09 \mathrm{E}-04$ \\
\hline & Systemic lupus erythematosus & 4 & $2.77 \mathrm{E}-03$ \\
\hline & Protein processing in endoplasmic reticulum & 4 & $5.55 \mathrm{E}-03$ \\
\hline & Graft-versus-host disease & 2 & $1.39 \mathrm{E}-02$ \\
\hline & Intestinal immune network for IgA production & 2 & $1.88 \mathrm{E}-02$ \\
\hline & JAK-STAT signaling pathway & 3 & $3.01 \mathrm{E}-02$ \\
\hline & Antigen processing and presentation & 2 & 4.40E-02 \\
\hline
\end{tabular}

${ }^{a}$ Number of differentially expressed genes that belong to these terms. 


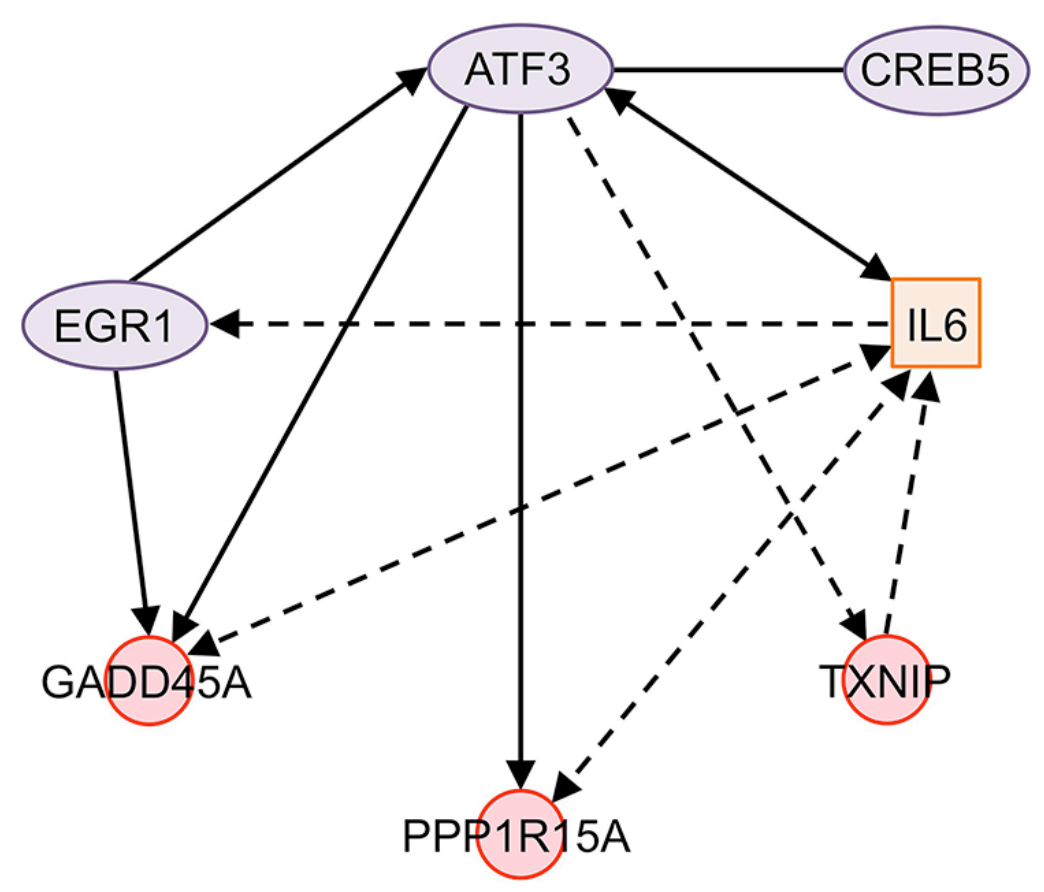

Figure 2: Network of ONC-regulated genes in NCI/ADR-RES cells. ATF3 directly promotes the expression of GADD45A, PPP1R15A, and IL6. It indirectly increases the expression of TXNIP and EGR1 and binds to CREB5. ATF3 and GADD45A expression is increased by EGR1. IL6 affects the expression of ATF3 and indirectly that of EGR1. At the same time, IL6 indirectly affects and is affected by PPP1R15A and GADD45A, and it is also indirectly affected by TXNIP. Solid line: interaction between two genes or the proteins coded by them; solid arrows: direct effect on gene expression; dotted arrows: indirect effect on gene expression; solid double arrows: reciprocal direct effect on gene expression; dotted double arrows: reciprocal indirect effect on gene expression, no physical contact between them or the proteins coded by them is required; ovals: transcription regulators; squares: cytokines; circles: other functions. Figure designed using the Ingenuity Pathway Analysis (IPA) package available online at http://www.ingenuity.com/products/ipa. Genes: ATF3, activation transcription factor 3; CREB5, cAMP responsive element binding protein 5; EGR1, early growth response 1; GADD45A, growth arrest and DNA damage-inducible, alpha; IL6, interleukin 6; PPP1R15A, protein phosphatase 1, regulatory subunit 15A; TXNIP, thioredoxin-interacting protein.

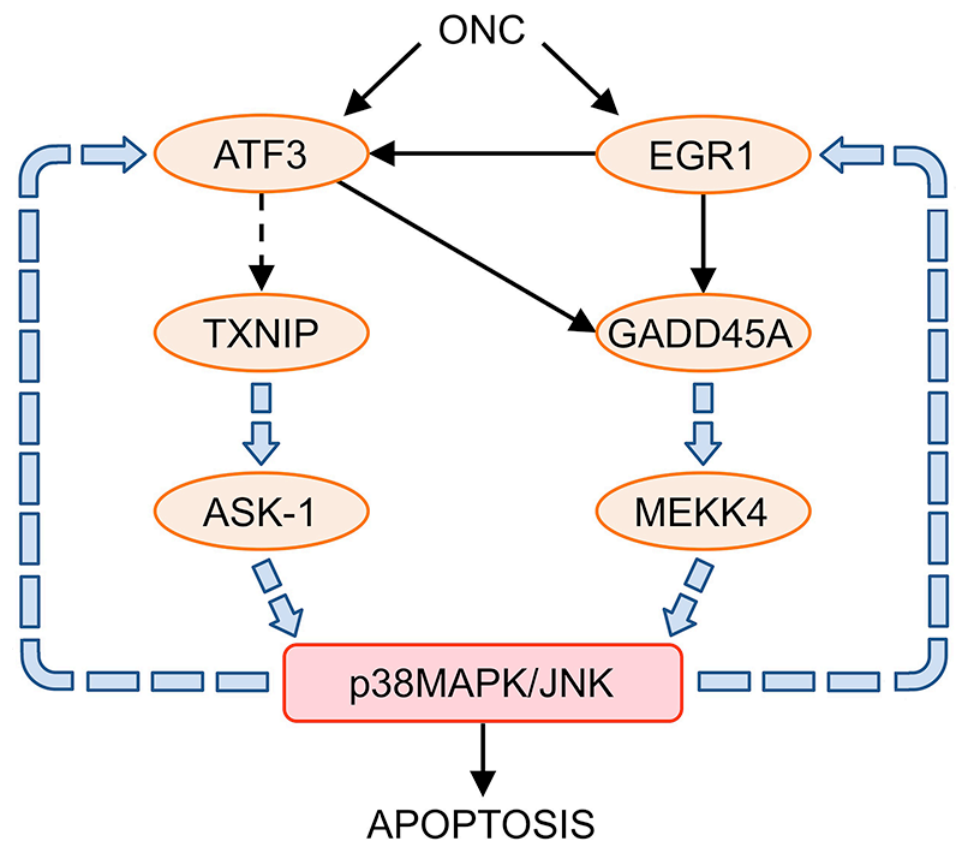

Figure 3: Relationship between ONC up-regulated genes and the p38MAPK/JNK signaling pathways. ONC up-regulated and interconnected ATF3, EGR1, TXNIP, and GADD45A genes, activate p38MAPK/JNK signaling pathways through ASK-1 and MEKK4, which leads to cell apoptosis. The activation of $38 \mathrm{MAPK} / \mathrm{JNK}$ reinforces the expression of the ONC up-regulated genes: ATF3 and EGR1. 
anti-tumorigenic abilities, depending on the cell type and conditions. It is also involved in metabolism control [76]. Its overexpression in cancer has been linked to cachexia [77], a drawback in the therapeutic use of ONC. Nevertheless, this effect may be partly counterbalanced by the ONC-induced overexpression of myocyte enhancer factor 2C (MEF2C), which is found among the 20 most ONC up-regulated genes (Table 1). MEF2C is a myogenic transcription factor that plays a critical role in skeletal muscle development and differentiation, but is down-regulated in cachexia [78]. In addition, ONC overexpression of inteleukin-33 (IL33), together with amphiregulin (AREG) (Table 1), may offset the pro-inflammatory properties of IL6 because it has been recently demonstrated that both participate in a pathway of innate immune cell-mediated tissue protection in the intestinal track [79]. Finally, ATF3 has been described as being able to epigenetically regulate the EGR1 expression, leading to attenuation of pro-inflammatory chemokines in human enterocytes [80].

\section{The mechanism described for $\mathrm{ONC}$ cytotoxicity is cell-line independent}

Six of the seven ONC differentially expressed genes found in the OVCAR- 8 cell line are also differentially expressed in the NCI/ADR-RES cell line (see the Results section). Additionally, in both cell lines, ATF3 and TXNIP are the most ONC up-regulated genes (see the Results section). Furthermore, in OVCAR-8 cell line a slight but significant increase in the expression of the rest of the networked transcription regulators differentially expressed in NCI/ADR-RES is observed, with fold change values between 1.8 and 1.5. These results indicate that the effect induced by $\mathrm{ONC}$ on both cell lines is similar. It is also noteworthy that ONC treatment of different hMM cell lines increased the expression of ATF3, GADD45A, and IL6, and involved the MAPK and Jak-STAT signaling pathways [12], suggesting that the cytotoxic mechanism described here for ONC is not cell-type specific.

\section{ONC causes different pleiotropic effects than those observed for PE5}

ONC and PE5, as RNA-damaging drugs, have pleiotropic effects. Focusing on the NCI/ADR-RES results, the pleiotropic effects caused by these RNases are clearly different. ONC affects a lower number of genes (11.5fold less) than PE5 [28]. Among them, the percentage of up- and down-regulated genes differs between the two RNase treatments. Of the ONC differentially expressed genes, $89 \%$ are up-regulated and only $11 \%$ are downregulated, while about half of the PE5 differentially expressed genes are up-regulated and half are downregulated [28]. This suggests that the ONC initial action is the activation of gene expression, in agreement with the results previously obtained for ONC-treated hMM cell lines [12]. Interestingly, only the expression of 10 genes was altered by both RNase treatments (see the Results section). Among those genes, asparagine synthetase (ASNS) and the phosphoglycerate dehydrogenase (PHGDH) coding genes are worth mentioning. Both are down-regulated by both RNases and are involved in amino acid metabolism, although we have not found any term related to amino acid metabolism either in gene ontology analysis or in the KEGG pathway annotation. PE5 down-regulates more genes related to amino acid biosynthesis than the two mentioned above. Its effect is actually to down-regulate this de novo biosynthesis that is significantly increased in cancer cells [28]. In addition, the most PE5 up-regulated gene, Homeobox containing 1 (HMBOX1), is also found among the 20 most ONC up-regulated genes. However, its function is not well known and is controversial [81]. Very likely, these coincidences are only the result of the pleiotropic effects of both RNases. Moreover, among the affected gene ontology terms, only five were common between ONC and PE5, and when compared the KEGG pathway annotation, no terms showed coincidence ([28] and Table 3 ).

The mechanism used by PE5 to kill cancer cells is primarily related to its ability to down-regulate the expression of genes that code for enzymes involved in the metabolic pathways deregulated in the cancer cells. This decrease in the expression levels of metabolic enzymes is assisted by the down- and up-regulation of several oncogenes and tumor suppressor genes, respectively, that down-regulate the expression of genes coding for these enzymes [28]. The only link between ONC regulated genes and cell metabolism is through the glucose uptake inhibitory effect of TXNIP and ARRDC4 $[82,83]$.

Taken together, our results indicate that the cytotoxic mechanisms exerted by ONC and PE5 on tumor cells are different, which is consistent with their different cellular localization and perhaps with their different RNA targets, cytosolic RNAs, and nuclear RNAs, respectively. Thus, it is tempting to anticipate a synergistic effect between RNases that exert their action in the cell cytosol and those that do it in the cell nucleus.

\section{ONC as antiviral agent beyond its nucleic acids degradation ability}

ONC has been described as having antiviral activity [25]. Due to the ability of wild type ONC and several ONC variants to cleave different types of RNA [10-15], as well as dsRNA [24], their antiviral properties have been mainly linked to degradation of viral genomes and/ or host-cell nucleic acids needed for viral replication $[25,84]$. However, the finding that ONC cell treatment promotes the up-regulation of ATF3 transcription factor independently of the cell type, leads to the proposal that other mechanisms can strengthen ONC antiviral action. ATF3 is a transcription factor whose role in cancer is clearly cellular-context dependent, but it seems essential for the maintenance of host defense mechanisms [34]. 
Genomic integration of HPV DNA, occurring in more than $90 \%$ of cervical cancers, results in viral E6 protein expression that can interact with a cellular ubiquitin ligase E6-associated protein (E6AP) and target the tumor suppressor p53 for ubiquitin-mediated proteolysis [85]. ATF3 interaction with viral protein E6 prevents p53 from ubiquitination and degradation, leading to expression of p53-target genes, cell cycle arrest, and apoptotic cell death. Thus, ATF3 is a repressor of the oncogenic viral protein E6 and blocks HPV-induced carcinogenesis [86]. Moreover, the ONC overexpressed gene that codes for protein tyrosine phosphatase receptor type R (PTPRR) has been linked to the inhibition of MAPK signaling through dephosphorylation of p44/42 MAPK, which inhibits AP-1 and the subsequent expression of the E6 and E7 HPV oncoproteins responsible for cervical cancer initiation and progression [87].

After infection at a portal entry, herpes simplex viruses (HSV) infect sensory nerve endings and are retrograde transported to the neuronal nucleus, where they establish a silent or latent state. It is not well known how these virulent viruses remain silent. However, it has recently been described that ATF3 plays a key role in the maintenance of the HSV in this latent state by increasing the expression of a non-coding RNA known as the latencyassociated transcript (LAT) [88].

Finally, ONC also inhibits $90-99.9 \%$ of human immunodeficiency virus type 1 (HIV-1) replication in $\mathrm{H} 9$ leukemia cells over a four-day incubation at concentrations not toxic to uninfected H9 cells [25]. HIV-1 transactivator protein Tat uses CREB to promote IL10 production. Although the importance of this for HIV pathogenesis is not clear, IL10 can inhibit HIV-1 replication in monocytes and macrophages, suggesting that Tat/CREB-induced IL10 production provides a negative feedback signal to prevent excess HIV-1 replication [89].

\section{CONCLUSIONS}

Our results explain the cytotoxic mechanism induced by ONC in treated cancer cells through a network of different ONC up-regulated genes. Among them, ATF3 upregulation plays a central role in the key events triggered by ONC that finally lead to apoptosis. This mechanism is cell-type independent. Up-regulation of ATF3 may also explain the antiviral properties of this RNase. ONCaffected genes are different from those affected by nucleardirected RNases. The pleiotropic effects of both types of RNases make them attractive as therapeutics to treat either cancer or virus-promoted diseases.

\section{MATERIALS AND METHODS}

\section{ONC expression and purification}

Construction of plasmid expressing ONC (pONC) has been previously described [90]. Recombinant
ONC was produced and purified from Escherichia coli BL21(DE3) cells transformed with pONC essentially as described in [91]. The molecular mass of ONC was confirmed by matrix-assisted laser desorption/ionization time-of-flight (MALDI-TOF) mass spectrometry at the Unitat cientificotècnica de suport, Institut de Recerca, Hospital Universitari Vall d'Hebron (Barcelona, Spain). ONC concentration was determined by ultraviolet spectroscopy using an extinction coefficient at $280 \mathrm{~nm}$ of $10470 \mathrm{M}^{-1} \mathrm{~cm}^{-1}$, calculated as reported [92].

\section{Cell lines and culture conditions}

NCI/ADR-RES human ovarian cancer MDR cell line (formerly MCF-7/ADR) [93] was a generous gift from Dr. Ramon Colomer of the Institut Català d'Oncologia de Girona, Hospital Universitari de Girona Dr. Josep Trueta (Girona, Spain). It was initially obtained from the American Type Culture Collection (ATCC) (Manassas, Virginia) and was used immediately after thawing. The OVCAR-8 human ovarian cancer cell line was obtained from the National Cancer Institute's DCTD tumor repository (Frederick, USA) and was used immediately after thawing. NCI/ADR-RES and OVCAR-8 cells were routinely grown at $37^{\circ} \mathrm{C}$ in a humidified atmosphere of $5 \% \mathrm{CO}_{2}$ in DMEM (Gibco, Germany) containing $1.84 \mu \mathrm{M}$ doxorubicin (Tedec-Meiji Farma, Spain) and RPMI (Gibco, Germany), respectively, supplemented with $10 \%$ fetal bovine serum (Gibco, Germany), $50 \mathrm{U} / \mathrm{ml}$ penicillin, and $50 \mu \mathrm{g} / \mathrm{ml}$ streptomycin (Gibco, Germany). Cells remained free of Mycoplasma and were propagated according to established protocols.

\section{Cell proliferation assay}

NCI/ADR-RES cells ( $10^{4}$ per well) and OVCAR-8 cells $\left(1.5 \times 10^{3}\right.$ per well $)$ were seeded into 96-well plates. After $24 \mathrm{~h}$ of incubation, cells were treated for 24,36 , or $48 \mathrm{~h}$ with various concentrations of $\mathrm{ONC}$, ranging from 0.001 to $10 \mu \mathrm{M}$. Sensitivity to RNase was determined by the 3-(4,5-dimethylthiazol-2-yl)2,5-diphenyltetrazolium bromide (MTT) method used according to the manufacturer's instructions (Sigma, USA). Data were collected by measuring the absorbance at $570 \mathrm{~nm}$ with a Synergy 4 multi-well plate reader (Biotek Instruments, USA). The $\mathrm{IC}_{5}, \mathrm{IC}_{10}$, and $\mathrm{IC}_{15}$ values represent the concentrations of $\mathrm{ONC}$ required to inhibit cell proliferation by 5,10 , and $15 \%$, respectively, and were calculated by interpolation of the obtained growth curves. Data were calculated as the mean \pm SD of three independent experiments conducted in triplicates.

\section{ONC treatment and RNA isolation}

NCI/ADR-RES cells ( 2 x $10^{5}$ per well) and OVCAR- 8 cells $\left(5 \times 10^{4}\right.$ per well) were seeded into 6-well plates. After $24 \mathrm{~h}$ of incubation, cells were treated for $36 \mathrm{~h}$ 
with a concentration of ONC that caused a $10 \%$ decrease in cell proliferation $(0.5 \mu \mathrm{M}$ ONC for NCI/ADR-RES cells and $0.06 \mu \mathrm{M}$ ONC for OVCAR- 8 cells). Cells were then harvested at $400 \mathrm{xg}$ for $5 \mathrm{~min}$ at $4{ }^{\circ} \mathrm{C}$ and washed twice with cold PBS. Total RNA was extracted using the mirVana miRNA isolation kit (Applied Biosystems/Ambion, USA) according to the manufacturer's instructions and stored at $-80^{\circ} \mathrm{C}$. Four independent preparations were performed for each cell line. The 260/280 $\mathrm{nm}$ absorbance ratio of each sample was checked using a NanoDrop ND-1000 spectrophotometer (Thermo Fisher Scientific, USA). RIN values were used to check RNA integrity using an Agilent 2100 bioanalyzer (Agilent Technologies, USA). The RIN value corresponds to the ratio of ribosomal band areas to total area of the electropherogram and the height of the $18 \mathrm{~S}$ peak [94].

\section{Gene expression microarray analysis}

Gene expression microarray experiments were performed at Bioarray, S.L. (Alacant, Spain) using the SurePrint G3 Human Gene Expression Microarray (Agilent Technologies, USA), a highdensity oligonucleotide microarray that contains 60,000 probes, corresponding to 27,958 Entrez Gene RNAs and 7,419 lncRNAs. Sample preparation and microarray processing procedures were done according to the TwoColor Microarray-Based Gene Expression Analysis v. 6.5 (Agilent Technologies, USA). Briefly, $200 \mathrm{ng}$ of total RNA were used to synthesize double-stranded cDNA with AffinityScript-Reverse Transcriptase and Oligo dTPromoter Primer. cDNA was simultaneously amplified and transcribed into cyanine 3- or cyanine 5-labeled cRNA employing T7 RNA polymerase in the presence of cyanine 3-CTP or cyanine 5-CTP. The labeled cRNA (antisense) was purified, evaluated using a NanoDrop ND1000 spectrophotometer (Thermo Fisher Scientific, USA) and hybridized to the oligonucleotide microarrays at $65^{\circ} \mathrm{C}$ for $17 \mathrm{~h}$. Microarrays were then washed and scanned on a G2565CA microarray scanner upgraded to a resolution of 2 micron (Agilent Technologies, USA). Data were extracted from the resulting TIFF-images using the Feature Extraction software v. 10.7 (Agilent Technologies, USA). Raw microarray data were statistically analyzed using the Marray, pcaMethods, Limma, and RankProd software packages from Bioconductor (www.bioconductor. org), which uses the $\mathrm{R}$ statistical environment and programming language. In particular, the non-specific signal was removed from the total intensity using the normexp background correction method with an offset of 20 [95]. Then intra-slide normalization was done using the Loess method [96] to make intensities consistent within each array, and inter-slide normalization was performed employing the quantiles method [97] to achieve consistency between arrays. After each of these analyses, a quality control analysis of microarray data (RG density plot, MA plot, and M boxplot) was performed. Following normalization, the RankProd method [98] was applied to identify differentially expressed genes. Genes were considered differentially expressed when they had a false discovery rate adjusted $p$-value $\leq 0.05$ and a fold change $\geq 2$ or $\leq-2$. Data have been deposited in NCBI's Gene Expression Omnibus repository [99] (http://www.ncbi.nih. gov/geo) and are available under the accession number: GSE75494.

\section{Gene ontology analysis and KEGG pathway annotation}

In the NCI/ADR-RES cell line, differentially expressed genes were characterized functionally with a hypergeometric test to find over-represented gene ontology terms in the three main broad ontologies (biological process, molecular function, and cellular component) (www.geneontology.org), and were also mapped to the Kyoto Encyclopedia of Genes and Genomes (KEGG) (www.kegg.jp), which assigns proteins to pathways, to find over-represented pathways. The analyses were done using the GOstats and RamiGO software packages from Bioconductor (www.bioconductor.org). A p-value cutoff of 0.05 was used. The network representation of linkages between ONC-differentially expressed genes was constructed and drawn using the Ingenuity Pathway Analysis (IPA) package available on-line at http://www. ingenuity.com/products/ipa.

\section{Quantitative reverse transcription PCR (RT- qPCR)}

mRNA expression of three ONC up-regulated genes (TXNIP, ATF3, and GADD45A) and two downregulated genes (PHGDH and ASNS) were examined by RT-qPCR. The same RNA samples used for microarrays analysis were used in this analysis. First, RNA samples were digested with DNase to prevent genomic contamination using the RNase-Free DNase Set (Qiagen, Germany) according to the manufacturer's instructions. They were evaluated using an Agilent 2100 bioanalyzer (Agilent Technologies, USA) and a NanoDrop ND-1000 spectrophotometer (Thermo Fisher Scientific, USA). Then, for each sample, $0.5 \mu \mathrm{g}$ of RNA was used to synthesize single-stranded cDNA with the High-Capacity cDNA Reverse Transcription Kit (Applied Biosystems, USA) following the manufacturer's instructions. Gene-specific forward and reverse primers for the selected genes were designed with Primer3 (http://primer3.ut.ee) and checked with NetPrimer (http://www.premierbiosoft.com/netprimer/). Primer sequences for ONC-regulated genes were: TXNIP (F: GCTTGCGGAGTGGCTAAAGT; R: CTCACCTGTTGGCTGGTCTTC), ATF3 (F: AGTGAGT GCTTCTGCCATCGT; R: TGACAAAGGGCGTCAGG 
TTAG), GADD45A (F: GAGAGCAGAAGACC GAAAGGA; R: CAGGCACAACACCACGTTATC), PHGDH (F: TATTGTTCGCTCTGCCACCA; R: TCATAACCAAGATGCCCTTCC) and ASNS (F: AAAGCCGAGGAGGAGAGTGA, R: GGTGGCAGAG ACAAGTAATAGGA). To select a constitutive gene as a reference for normalizing data, the transcription abundances of five genes (ACTB, GUSB, TBP, HPRT1, and ALAS1) were measured for all cDNA samples. Primer sequences are described in reference [28]. Among them, TBP showed the highest stability (lower standard deviation of the $\mathrm{Ct}$; results not shown) and it was therefore selected for data normalization. Real-time PCRs were performed in an optical 96-well plate with an ABI PRISM 7300 Sequence Detector System (Applied Biosystems, USA), using SYBR Green to monitor dscDNA synthesis. Reactions contained 1x Power SYBR Green PCR Master Mix (Applied Biosystems, USA), $300 \mathrm{nM}$ of gene-specific forward primer, 300 $\mathrm{nM}$ of gene-specific reverse primer, and $5 \mu \mathrm{l}$ of a 50 fold dilution of the previously synthesized cDNA in a final volume of $20 \mu \mathrm{l}$. The following standard thermal profile was used for all real-time PCRs: $95^{\circ} \mathrm{C}$ for 10 min, 40 cycles of $95^{\circ} \mathrm{C}$ for $15 \mathrm{~s}$, and $60^{\circ} \mathrm{C}$ for $1 \mathrm{~min}$. A dissociation step was performed after amplification to confirm the presence of a single amplicon. To estimate variation in the technique, three technical replicates were carried out for each cDNA sample. Data were analyzed with the 7300 SDS 1.3.1 software (Applied Biosystems, USA). To generate a baseline-subtracted plot of the logarithmic increase in fluorescence signal $(\Delta R n)$ versus cycle number, baseline data were collected between cycles 3 and 15. All amplification plots were analyzed with an $\mathrm{Rn}$ threshold of 0.2 to obtain threshold cycle $\mathrm{Ct}$ ) values. The amplification efficiency for each gene was calculated based on five dilutions of cDNA ranging from 1 to $3.2 \times 10^{-4}$ and the equation $\mathrm{E}=10^{(-1 / \mathrm{slope})}$. All genes had an efficiency value between 1.85 and 2.05. The relative transcription abundances (RTA) of ONCtarget genes (TXNIP, ATF3, GADD45A, PHGDH, and ASNS) were calculated as RTA $=\mathrm{E}^{\Delta \mathrm{Ct} \text { (control-sample) }}{ }_{\text {(Target) }} /$ $\mathrm{E}^{\Delta \mathrm{Ct}(\text { control-sample) }}{ }_{\text {(Reference) }}[100]$, where control refers to a mix of equal amounts of untreated samples. Fold changes were calculated as the ratio between RTA values obtained for ONC-treated cells and those obtained for untreated cells. The absence of genomic DNA contamination was checked using non-retrotranscriptase controls and the absence of environmental contamination using nontemplate controls.

\section{ACKNOWLEDGMENTS}

We are very grateful to Dr. M. Figueras, Dr. O. Serra, and Mr. P. Boher from the Department of Biology, Universitat de Girona, for their help with the RT-qPCR experiments.

\section{CONFLICTS OF INTEREST}

The authors declare no potential conflict of interests.

\section{GRANT SUPPORT}

This work has been supported by grants BFU200906935 and BIO2013-43517 from Ministerio de Economia y Competitividad (Spain) and SING12/0 from UdG (Spain). A. V. acknowledges a predoctoral FPU fellowship from Ministerio de Educación y Ciencia (Spain).

\section{REFERENCES}

1. Arnold U, Schulenburg C, Schmidt D, Ulbrich-Hofmann R. Contribution of structural peculiarities of onconase to its high stability and folding kinetics. Biochemistry. 2006; 45:3580-3587.

2. Rutkoski T, Raines R. Evasion of ribonuclease inhibitor as a determinant of ribonuclease cytotoxicity. Current Pharmarmaceutical Biotechnology. 2008; 9:185-199.

3. Darzynkiewicz Z, Carter SP, Mikulski SM, Ardelt WJ, Shogen K. Cytostatic and cytotoxic effects of Pannon (P-30 Protein), a novel anticancer agent. Cell Proliferation. 1988; 21:169-182.

4. Beck AK, Pass HI, Carbone M, Yang H. Ranpirnase as a potential antitumor ribonuclease treatment for mesothelioma and other malignancies. Future Oncology. 2008; 4:341-349.

5. Ardelt W, Shogen K, Darzynkiewicz Z. Onconase and amphinase, the antitumor ribonucleases from Rana pipiens oocytes. Current Pharmarmaceutical Biotechnology. 2008; 9:215-225.

6. Ribó M, Benito A, Vilanova M. Antitumor ribonucleases in Ribonucleases. Nucleic Acids Molecular Biolology (Nicholson AW, ED.) Springer Berlin Heidelberg; 2011. Vol 26. pp 55-88.

7. Costanzi J, Sidransky D, Navon A, Goldsweig H. Ribonucleases as a novel pro-apoptotic anticancer strategy: review of the preclinical and clinical data for ranpirnase. Cancer Investigation. 2005; 23:643-650.

8. Porta C, Paglino C, Mutti L. Ranpirnase and its potential for the treatment of unresectable malignant mesothelioma. Biologics. 2008; 2:601-609.

9. Pavlakis N, Vogelzang NJ. Ranpirnase-an antitumour ribonuclease: its potential role in malignant mesothelioma. Expert Opinion on Biological Therapy. 2006; 6:391-399.

10. Wu Y, Mikulski SM, Ardelt W, Rybak SM, Youle RJ. A cytotoxic ribonuclease. Study of the mechanism of onconase cytotoxicity. The Journal of Biological Chemistry. 1993; 268:10686-10693.

11. Juan G, Ardelt B, Li X, Mikulski SM, Shogen K, Ardelt W, Mittelman A, Darzynkiewicz Z. G(1) arrest of U937 cells by onconase as associated with suppression of cyclin 
D3 expression, induction of p16(INK4A), P21(WAF1/ CIP1) and p27(KIP) and decreased pRb phosphorylation. Leukemia. 1998; 12:1241-1248.

12. Altomare DA, Rybak SM, Pei J, Maizel J V, Cheung M, Testa JR, Shogen K. Onconase responsive genes in human mesothelioma cells: implications for an RNA damaging therapeutic agent. BMC Cancer. 2010; 10:34.

13. Saxena SK, Sirdeshmukh R, Ardelt W, Mikulski SM, Shogen K, Youle RJ. Entry into cells and selective degradation of tRNAs by a cytotoxic member of the RNase A family. The Journal of Biological Chemistry. 2002; 277:15142-15146.

14. Ardelt B, Ardelt W, Darzynkiewicz Z. Cytotoxic Ribonucleases and RNA Interference (RNAi). Cell Cycle. 2003; 2:22-24.

15. Zhao H, Ardelt B, Ardelt W, Shogen K, Darzynkiewicz Z. The cytotoxic ribonuclease onconase targets RNA interference (siRNA). Cell Cycle. 2008; 7:3258-3261.

16. Qiao M, Zu L-D, He X-H, Shen R-L, Wang Q-C, Liu M-F. Onconase downregulates microRNA expression through targeting microRNA precursors. Cell Research. 2012; 22:1199-1202.

17. Tsai SY, Ardelt B, Hsieh T-C, Darzynkiewicz Z, Shogen $\mathrm{K}$, Wu JM. Treatment of Jurkat acute T-lymphocytic leukemia cells by onconase (Ranpirnase) is accompanied by an altered nucleocytoplasmic distribution and reduced expression of transcription factor NF- $\mathrm{BB}$. International Journal of Oncology. 2004; 25:1745-1752.

18. Iordanov MS, Wong J, Newton DL, Rybak SM, Bright RK, Flavell RA, Davis RJ, Magun BE. Differential requirement for the stress-activated protein kinase/c-Jun $\mathrm{NH}(2)$-terminal kinase in RNAdamage-induced apoptosis in primary and in immortalized fibroblasts. Molecular Cell Biology Research Communications. 2000; 4:122-128.

19. Grabarek J, Ardelt B, Du L, Darzynkiewicz Z. Activation of caspases and serine proteases during apoptosis induced by onconase (Ranpirnase). Experimental Cell Research. 2002; 278: 61-71.

20. Ardelt B, Ardelt W, Pozarowski P, Kunicki J, Shogen K, Darzynkiewicz Z. Cytostatic and cytotoxic properties of amphinase: A novel cytotoxic ribonuclease from Rana pipiens oocytes. Cell Cycle. 2007; 6:3097-3102.

21. Mei Y, Yong J, Liu H, Shi Y, Meinkoth J, Dreyfuss G, Yang X. tRNA binds to cytochrome $\mathrm{c}$ and inhibits caspase activation. Molecular Cell. 2010; 37:668-678.

22. Michaelis M, Cinatl J, Anand P, Rothweiler F, Kotchetkov R, Deimling A Von, Doerr HW, Shogen K, Cinatl J. Onconase induces caspase-independent cell death in chemoresistant neuroblastoma cells. Cancer Letters. 2007; 250:107-116.

23. Fiorini C, Cordani M, Gotte G, Picone D, Donadelli M. Onconase induces autophagy sensitizing pancreatic cancer cells to gemcitabine and activates Akt/mTOR pathway in a ROS-dependent manner. Biochimimica et Biophysica Acta. 2015; 1853:549-560.
24. Saxena A, Saxena SK, Shogen K. Effect of Onconase on double-stranded RNA in vitro. Anticancer Research. 2009; 29:1067-1071.

25. Youle RJ, Wu YN, Mikulski SM, Shogen K, Hamilton RS, Newton D, D'Alessio G, Gravell M. RNase inhibition of human immunodeficiency virus infection of H9 cells. Proceedings of the National Academy of Sciences of the United States of America. 1994; 91:6012-6016.

26. Saxena SK, Ardelt W inventors; Tamir Biotechnology, Inc., assignee. Methods of treating human papillomavirus. United States patent US 8663964 B2. 2014 Mar 4.

27. Saxena SK, Ardelt W, inventors; Tamir Biotechnology, Inc., assignee. Methods of treating infections originating from viruses in the herpesviridae family. United States patent US 8518399 B2. 2013.

28. Vert A, Castro J, Ribó M, Benito A, Vilanova M. A nucleardirected human pancreatic ribonuclease (PE5) targets the metabolic phenotype of cancer cells. Oncotarget. 2016; 7: 18309-18324. doi: 10.18632/oncotarget.7579.

29. Bosch M, Benito A, Ribó M, Puig T, Beaumelle B, Vilanova M. A Nuclear Localization Sequence Endows Human Pancreatic Ribonuclease with Cytotoxic Activity. Biochemistry. 2004; 43:2167-2177.

30. Tubert P, Rodríguez M, Ribó M, Benito A, Vilanova M. The nuclear transport capacity of a human-pancreatic ribonuclease variant is critical for its cytotoxicity. Investigational New Drugs. 2011; 29:811-817.

31. Castro J, Ribó M, Navarro S, Nogués MV, Vilanova $\mathrm{M}$, Benito A. A human ribonuclease induces apoptosis associated with p21WAF1/CIP1 induction and JNK inactivation. BMC Cancer. 2011; 11:9.

32. Kholodnyuk ID, Kozireva S, Kost-Alimova M, Kashuba $\mathrm{V}$, Klein G, Imreh S. Down regulation of $3 p$ genes, LTF, SLC38A3 and DRR1, upon growth of human chromosome 3-mouse fibrosarcoma hybrids in severe combined immunodeficiency mice. International Journal of Cancer. 2006; 119:99-107.

33. Kantyka T, Fischer J, Wu Z, Declercq W, Reiss K, Schröder $\mathrm{J}-\mathrm{M}$, Meyer-Hoffert U. Inhibition of kallikrein-related peptidases by the serine protease inhibitor of Kazal-type 6 . Peptides. 2011; 32:1187-1192.

34. Thompson MR, Xu D, Williams BRG. ATF3 transcription factor and its emerging roles in immunity and cancer. Journal of Molecular Medicine. 2009; 87:1053-1060.

35. Syed V, Mukherjee K, Lyons-Weiler J, Lau K-M, Mashima T, Tsuruo T, Ho S. Identification of ATF-3, caveolin-1, DLC-1, and NM23-H2 as putative antitumorigenic, progesterone-regulated genes for ovarian cancer cells by gene profiling. Oncogene. 2005; 24:1774-1787.

36. Huang X, Li X, Guo B. KLF6 induces apoptosis in prostate cancer cells through up-regulation of ATF3. The Journal of Biological Chemistry. 2008; 283:29795-29801. 
37. Mashima $\mathrm{T}$, Udagawa $\mathrm{S}$, Tsuruo $\mathrm{T}$. Involvement of transcriptional repressor ATF3 in acceleration of caspase protease activation during DNA damaging agent-induced apoptosis. Journal of Cellular Physiology. 2001; 188:352-358.

38. Yan C, Aggarwal BB, Jamaluddin MS, Myers J, Boyd DD. Gene expression profiling identifies activating transcription factor 3 as a novel contributor to the proapoptotic effect of curcumin. Molecular Cancer Therapeutics. 2005; $4: 233-241$.

39. O'Brien A, Barber JEB, Reid S, Niknejad N, Dimitroulakos J. Enhancement of cisplatin cytotoxicity by disulfiram involves activating transcription factor 3. Anticancer Research 2012; 32:2679-2688.

40. Niknejad N, Gorn-Hondermann I, Ma L, Zahr S, JohnsonObeseki S, Corsten M, Dimitroulakos J. Lovastatin-induced apoptosis is mediated by activating transcription factor 3 and enhanced in combination with salubrinal. International Journal of Cancer. 2014; 134:268-279.

41. Jan YH, Tsai HY, Yang CJ, Huang MS, Yang YF, Lai TC, Lee CH, Jeng YM, Huang CY, Su JL, Chuang YJ, Hsiao M. et al. Adenylate kinase- 4 is a marker of poor clinical outcomes that promotes metastasis of lung cancer by downregulating the transcription factor ATF3. Cancer Research 2012; 72:5119-5129.

42. Yuan X, Yu L, Li J, Xie G, Rong T, Zhang L, Chen J, Meng Q, Irving AT, Wang D, Williams ED, Liu JP, Sadler AJ, et al. ATF3 suppresses metastasis of bladder cancer by regulating gelsolin-mediated remodeling of the actin cytoskeleton. Cancer Research. 2013; 73:3625-3637.

43. Xie J-J, Xie Y-M, Chen B, Pan F, Guo J-C, Zhao Q, Shen J-H, Wu Z-Y, Xu J-Y, Li E-M. ATF3 functions as a novel tumor suppressor with prognostic significance in esophageal squamous cell carcinoma. Oncotarget. 2014; 5:8569-8582. doi: 10.18632/oncotarget.2322.

44. Xu K, Zhou Y, Qiu W, Liu X, Xia M, Liu L, Liu X, Zhao D, Wang Y. Activating transcription factor 3 (ATF3) promotes sublytic C5b-9-induced glomerular mesangial cells apoptosis through up-regulation of Gadd $45 \alpha$ and KLF6 gene expression. Immunobiology. 2011; 216:871-881.

45. Jiang H-Y, Wek SA, McGrath BC, Lu D, Hai T, Harding HP, Wang X, Ron D, Cavener DR, Wek RC. Activating transcription factor 3 is integral to the eukaryotic initiation factor 2 kinase stress response. Molecular and Cellular Biology. 2004; 24:1365-1377.

46. Qi W, Chen X, Holian J, Tan CYR, Kelly DJ, Pollock CA. Transcription Factors Krüppel-Like Factor 6 and Peroxisome Proliferator-Activated Receptor- $\gamma$ Mediate High Glucose-Induced Thioredoxin-Interacting Protein. The American Journal of Pathology. 2009; 175:1858-1867.

47. Newman JRS, Keating AE. Comprehensive Identification of Human bZIP Interactions with Coiled-Coil Arrays. Science. 2003; 300:2097-2101.

48. Bottone FG, Moon Y, Alston-Mills B, Eling TE. Transcriptional regulation of activating transcription factor 3 involves the early growth response- 1 gene. The Journal of
Pharmacology and Experimental Therapeutics. 2005; 315: 668-677.

49. Thyss R, Virolle V, Imbert V, Peyron J-F, Aberdam D, Virolle T. NF-kappaB/Egr-1/Gadd45 are sequentially activated upon UVB irradiation to mediate epidermal cell death. The EMBO Journal. 2005; 24:128-137.

50. Levin WJ, Press MF, Gaynor RB, Sukhatme VP, Boone TC, Reissmann PT, Figlin RA, Holmes EC, Souza LM, Slamon DJ. Expression patterns of immediate early transcription factors in human non-small cell lung cancer. The Lung Cancer Study Group. Oncogene. 1995; 11:1261-1269.

51. Huang RP, Fan Y, de Belle I, Niemeyer C, Gottardis MM, Mercola D, Adamson ED. Decreased Egr-1 expression in human, mouse and rat mammary cells and tissues correlates with tumor formation. Inernational Journal of Cancer. 1997; 72:102-109.

52. Calogero A, Arcella A, De Gregorio G, Porcellini A, Mercola D, Liu C, Lombari V, Zani M, Giannini G, Gagliardi FM, Caruso R, Gulino A, Frati L, et al. The early growth response gene EGR-1 behaves as a suppressor gene that is down-regulated independent of ARF/Mdm2 but not p53 alterations in fresh human gliomas. Clinical Cancer Research. 2001; 7:2788-2796.

53. Zagurovskaya M, Shareef MM, Das A, Reeves A, Gupta S, Sudol M, Bedford MT, Prichard J, Mohiuddin M, Ahmed MM. EGR-1 forms a complex with YAP-1 and upregulates Bax expression in irradiated prostate carcinoma cells. Oncogene. 2009; 28:1121-1131.

54. Iordanov MS, Ryabinina OP, Wong J, Dinh TH, Newton DL, Rybak SM, Magun BE. Molecular determinants of apoptosis induced by the cytotoxic ribonuclease onconase: Evidence for cytotoxic mechanisms different from inhibition of protein synthesis. Cancer Research 2000; 60:1983-1994.

55. Siafakas RA, Richardson DR. Growth arrest and DNA damage-45 alpha (GADD45 $\alpha$ ). International Journal of Biochemistry and Cell Biology. 2009; 41:986-989.

56. Wang XW, Zhan Q, Coursen JD, Khan MA, Kontny HU, Yu L, Hollander MC, O'Connor PM, Fornace AJ, Harris CC. GADD45 induction of a G2/M cell cycle checkpoint. Proceedings of the National Academy of Sciences of the United States of America. 1999; 96:3706-3711.

57. Zhan Q, Antinore MJ, Wang XW, Carrier F, Smith ML, Harris CC, Fornace AJ. Association with Cdc2 and inhibition of $\mathrm{Cdc} 2 / \mathrm{Cyclin} \mathrm{B} 1$ kinase activity by the p53regulated protein Gadd45. Oncogene. 1999; 18:2892-2900.

58. Liu L, Ito S, Nishio N, Sun Y, Chen N, Tanaka Y, Isobe K-I. GADD34 Facilitates Cell Death Resulting from Proteasome Inhibition. Anticancer Research. 2015; 35:5317-5324.

59. Zerbini LF, Wang Y, Czibere A, Correa RG, Cho J-Y, Ijiri K, Wei W, Joseph M, Gu X, Grall F, Goldring MB, Zhou J-R, Libermann TA, et al. NF-kappa B-mediated repression of growth arrest- and DNA-damage-inducible proteins 45alpha and gamma is essential for cancer cell survival. Proceedings of the National Academy of Sciences of the United States of America. 2004; 101:13618-13623. 
60. Nasu M, Carbone M, Gaudino G, Ly BH, Bertino P, Shimizu D, Morris P, Pass HI, Yang H. Ranpirnase Interferes with NF- $\kappa \mathrm{B}$ Pathway and MMP9 Activity, Inhibiting Malignant Mesothelioma Cell Invasiveness and Xenograft Growth. Genes Cancer. 2011; 2:576-84. doi: $10.1177 / 1947601911412375$.

61. Goparaju CM, Blasberg JD, Volinia S, Palatini J, Ivanov S, Donington JS, Croce C, Carbone M, Yang H, Pass HI. Onconase mediated NFK $\beta$ downregulation in malignant pleural mesothelioma. Oncogene. 2011; 30:2767-2777.

62. Junn E, Han SH, Im JY, Yang Y, Cho EW, Um HD, Kim DK, Lee KW, Han PL, Rhee SG, Choi I. Vitamin D3 up-regulated protein 1 mediates oxidative stress via suppressing the thioredoxin function. Journal of Immunology. 2000; 164:6287-6295.

63. Ikarashi M, Takahashi Y, Ishii Y, Nagata T, Asai S, Ishikawa K. Vitamin D3 up-regulated protein 1 (VDUP1) expression in gastrointestinal cancer and its relation to stage of disease. Anticancer Research. 2002; 22:4045-4048.

64. Han SH, Jeon JH, Ju HR, Jung U, Kim KY, Yoo HS, Lee YH, Song KS, Hwang HM, Na YS, Yang Y, Lee KN, Choi I. VDUP1 upregulated by TGF-betal and 1,25-dihydorxyvitamin D3 inhibits tumor cell growth by blocking cell-cycle progression. Oncogene. 2003; 22:4035-4046.

65. Tome ME, Johnson DBF, Rimsza LM, Roberts RA, Grogan TM, Miller TP, Oberley LW, Briehl MM. A redox signature score identifies diffuse large B-cell lymphoma patients with a poor prognosis. Blood. 2005; 106:3594-3601.

66. Nishizawa K, Nishiyama H, Matsui Y, Kobayashi T, Saito R, Kotani H, Masutani H, Oishi S, Toda Y, Fujii N, Yodoi J, Ogawa O. Thioredoxin-interacting protein suppresses bladder carcinogenesis. Carcinogenesis. 2011; 32:1459-1466.

67. Sheth SS, Bodnar JS, Ghazalpour A, Thipphavong CK, Tsutsumi S, Tward AD, Demant P, Kodama T, Aburatani H, Lusis AJ. Hepatocellular carcinoma in Txnip-deficient mice. Oncogene. 2006; 25:3528-3536.

68. Goldberg SF, Miele ME, Hatta N, Takata M, PaquetteStraub C, Freedman LP, Welch DR. Melanoma metastasis suppression by chromosome 6: evidence for a pathway regulated by CRSP3 and TXNIP. Cancer Research. 2003; 63:432-440.

69. Jeon J-H, Lee K-N, Hwang CY, Kwon K-S, You K-H, Choi I. Tumor suppressor VDUP1 increases p27(kip1) stability by inhibiting JAB1. Cancer Research. 2005; 65:4485-4489.

70. Nishinaka Y, Nishiyama A, Masutani H, Oka S, Ahsan KM, Nakayama Y, Ishii Y, Nakamura H, Maeda M, Yodoi J. Loss of thioredoxin-binding protein-2/vitamin D3 up-regulated protein 1 in human T-cell leukemia virus type I-dependent T-cell transformation: implications for adult T-cell leukemia leukemogenesis. Cancer Research. 2004; 64: 1287-1292.

71. Chen CL, Lin CF, Chang WT, Huang WC, Teng CF, Lin YS. Ceramide induces p38 MAPK and JNK activation through a mechanism involving a thioredoxin-interacting protein-mediated pathway. Blood. 2008; 111:4365-4374.
72. Takekawa M, Saito H. A Family of Stress-Inducible GADD45-like Proteins Mediate Activation of the StressResponsive MTK1/MEKK4 MAPKKK. Cell. 1998; 95:521-530.

73. Lim CP, Jain N, Cao X. Stress-induced immediate-early gene, egr-1, involves activation of p38/JNK1. Oncogene. 1998; 16:2915-2926.

74. Rolli M, Kotlyarov A, Sakamoto KM, Gaestel M, Neininger A. Stress-induced stimulation of early growth response gene- 1 by $\mathrm{p} 38 /$ stress-activated protein kinase 2 is mediated by a cAMP-responsive promoter element in a MAPKAP kinase 2-independent manner. The Journal of Biological Chemistry. 1999; 274:19559-19564.

75. Lu D, Chen J, Hai T. The regulation of ATF3 gene expression by mitogen-activated protein kinases. Biochemical Journal. 2007; 401:559-467.

76. Mauer J, Denson JL, Brüning JC. Versatile functions for IL-6 in metabolism and cancer. Trends in Immunology. 2015; 36:92-101.

77. Yasumoto K, Mukaida N, Harada A, Kuno K, Akiyama M, Nakashima E, Fujioka N, Mai M, Kasahara T, FujimotoOuchi K, Mori K, Tanaka Y, Matsushima K. Molecular Analysis of the Cytokine Network Involved in Cachexia in Colon 26 Adenocarcinoma-bearing Mice. Cancer Research. 1995; 55:921-927.

78. Lecker SH, Jagoe RT, Gilbert A, Gomes M, Baracos V, Bailey J, Price SR, Mitch WE, Goldberg AL. Multiple types of skeletal muscle atrophy involve a common program of changes in gene expression. FASEB Journal. 2004; 18:39-51.

79. Monticelli LA, Osborne LC, Noti M, Tran SV, Zaiss DMW, Artis DA. IL-33 promotes an innate immune pathway of intestinal tissue protection dependent on amphiregulinEGFR interactions. Proceedings of the National Academy of Sciences of the United States of America. 2015; 112:10762-10767.

80. Park S-H, Do KH, Choi HJ, Kim J, Kim K-H, Park J, Oh CG, Moon Y. Novel regulatory action of ribosomal inactivation on epithelial Nod2-linked proinflammatory signals in two convergent ATF3-associated pathways. Journal of Immunology. 2013; 191:5170-5181.

81. Ma H, Su L, Yue H, Yin X, Zhao J, Zhang S, Kung H, Xu Z, Miao J. HMBOX1 interacts with MT2A to regulate autophagy and apoptosis in vascular endothelial cells. Scientific Reports. 2015; 5:15121.

82. Elgort MG, O'Shea JM, Jiang Y, Ayer DE. Transcriptional and Translational Downregulation of Thioredoxin Interacting Protein Is Required for Metabolic Reprogramming during G(1). Genes Cancer. 2010; 1:893907. doi: 10.1177/1947601910389604.

83. Patwari P, Chutkow WA, Cummings K, Verstraeten VLRM, Lammerding J, Schreiter ER, Lee RT. Thioredoxinindependent regulation of metabolism by the alpha-arrestin proteins. The Journal of Biological Chemistry. 2009; 284:24996-25003. 
84. Suhasini AN, Sirdeshmukh R. Onconase action on tRNALys3, the primer for HIV-1 reverse transcription. Biochemical and Biophysical Research Communications. 2007; 363:304-309.

85. Schiffman M, Castle PE, Jeronimo J, Rodriguez AC, Wacholder S. Human papillomavirus and cervical cancer. Lancet. 2007; 370:890-907.

86. Wang H, Mo P, Ren S, Yan C. Activating transcription factor 3 activates $\mathrm{p} 53$ by preventing E6-associated protein from binding to E6. The Journal of Biological Chemistry. 2010; 285:13201-13210.

87. Su P-H, Lin Y-W, Huang R-L, Liao Y-P, Lee H-Y, Wang H-C, Chao T-K, Chen C-K, Chan MWY, Chu T-Y, Yu M-H, Lai H-C. Epigenetic silencing of PTPRR activates MAPK signaling, promotes metastasis and serves as a biomarker of invasive cervical cancer. Oncogene. 2013; 32:15-26.

88. Shu M, Du T, Zhou G, Roizman B. Role of activating transcription factor 3 in the synthesis of latency-associated transcript and maintenance of herpes simplex virus 1 in latent state in ganglia. Proceedings of the National Academy of Sciences of the United States of America. 2015; 112:E5420-E5426.

89. Wen AY, Sakamoto KM, Miller LS. The role of the transcription factor CREB in immune function. Journal of Immunology. 2010; 185:6413-6419.

90. Leland PA, Schultz LW, Kim B-M, Raines RT. Ribonuclease A variants with potent cytotoxic activity. Proceedings of the National Academy of Sciences of the United States of America. 1998; 95:10407-10412.

91. Ribó M, Bosch M, Torrent G, Benito A, Beaumelle B, Vilanova M. Quantitative analysis, using MALDI-TOF mass spectrometry, of the N-terminal hydrolysis and cyclization reactions of the activation process of onconase. European Journal of Biochemistry. 2004; 271:1163-1171.
92. Pace CN, Vajdos F, Fee L, Grimsley G, Gray T. How to measure and predict the molar absorption coefficient of a protein. Protein Science. 1995; 4:2411-2423.

93. Liscovitch M, Ravid D. A case study in misidentification of cancer cell lines: MCF-7/AdrR cells (re-designated NCI/ADR-RES) are derived from OVCAR-8 human ovarian carcinoma cells. Cancer Letters. 2007; 245:350-352.

94. Viljoen KS, Blackburn JM. Quality assessment and data handling methods for Affymetrix Gene 1.0 ST arrays with variable RNA integrity. BMC Genomics. 2013; 14:14.

95. Ritchie ME, Silver J, Oshlack A, Holmes M, Diyagama D, Holloway A, Smyth GK. A comparison of background correction methods for two-colour microarrays. Bioinformatics. 2007; 23:2700-2707.

96. Cleveland W, Devlin S. Locally Weighted Regression: An Approach to Regression Analysis by Local Fitting. Journal of the American Statistical Association. 1988; 83:596-610.

97. Bolstad BM, Irizarry RA, Astrand M, Speed TP. A comparison of normalization methods for high density oligonucleotide array data based on variance and bias. Bioinformatics. 2003; 19:185-193.

98. Breitling R, Armengaud P, Amtmann A, Herzyk P. Rank products: a simple, yet powerful, new method to detect differentially regulated genes in replicated microarray experiments. FEBS Letters. 2004; 573:83-92.

99. Edgar R, Domrachev M, Lash AE. Gene Expression Omnibus: NCBI gene expression and hybridization array data repository. Nucleic Acids Research. 2002; 30:207-210.

100. Pfaffl MW, Pfaffl MW. A new mathematical model for relative quantification in real-time RT-PCR. Nucleic Acids Research. 2001; 29:e45. 